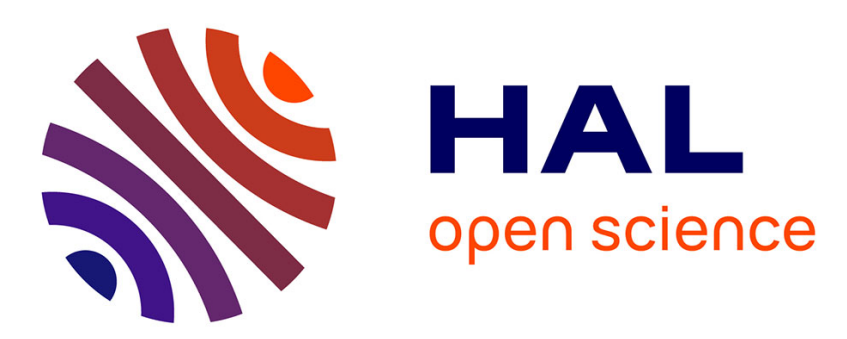

\title{
TCTP Has a Crucial Role in the Different Stages of Prostate Cancer Malignant 4 Progression 5
}

Virginie Baylot, Sara Karaki, Palma Rocchi

\section{To cite this version:}

Virginie Baylot, Sara Karaki, Palma Rocchi. TCTP Has a Crucial Role in the Different Stages of Prostate Cancer Malignant 4 Progression 5. TCTP/tpt1 - Remodeling Signaling from Stem Cell to Disease, 64, 2017, Results and Problems in Cell Differentiation, 10.1007/978-3-319-67591-6_13 . hal-01790677

\section{HAL Id: hal-01790677 \\ https://hal.science/hal-01790677}

Submitted on 13 May 2018

HAL is a multi-disciplinary open access archive for the deposit and dissemination of scientific research documents, whether they are published or not. The documents may come from teaching and research institutions in France or abroad, or from public or private research centers.
L'archive ouverte pluridisciplinaire HAL, est destinée au dépôt et à la diffusion de documents scientifiques de niveau recherche, publiés ou non, émanant des établissements d'enseignement et de recherche français ou étrangers, des laboratoires publics ou privés. 


\title{
Chapter 13 \\ TCTP Has a Crucial Role in the Different \\ Stages of Prostate Cancer Malignant \\ Progression
}

\begin{abstract}
Prostate cancer (PC) is the second most common cause of cancer-related 6 mortality in men in the western world after lung cancer. Many patients are not 7 candidates for resection given the advanced stage of their cancer. The primary 8 treatment for advanced PC is the castration therapy which supresses the production 9 of androgens, hormone that promotes PC growth. Despite the efficiency of the 10 castration therapy, most patients develop castration resistant disease which remains 11 uncurable. Clearly, novel approaches are required to effectively treat castration 12 resistant PC (CRPC). New strategies that identify the molecular mechanisms by 13 which PC becomes resistant to conventional therapies may enable the identification 14 of novel therapeutic targets that could improve clinical outcome. Recent studies 15 have demonstrated the implication of TCTP's over-expression in PC and CRPC, 16 and its role in resistance to treatment. TCTP's interaction with p53 and their 17 negative feedback loop regulation have also been described to be causal for PC 18 progression and invasion. A novel nanotherapy that inhibits TCTP has been devel- 19 oped as a new therapeutical strategy in CRPC. This chapter will highlight the role of 20 TCTP as new therapeutic target in PC, in particular, therapy-resistant advanced PC 21 and report the development of novel nanotherapy against TCTP that restore treat- 22 ment-sensitivity in CRPC that deserve to be tested in clinical trial. 23

\footnotetext{
V. Baylot Medicine, Stanford, CA 94305, USA

S. Karaki $\bullet$ P. Rocchi $(\bowtie)$

France

Institut Paoli-Calmettes, Marseille 13009, France

Aix-Marseille Université, 13284 Marseille, France

CNRS, UMR7258, CRCM, Marseille 13009, France

e-mail:palma.rocchi@inserm.fr
}

Division of Oncology, Departments of Medicine and Pathology, Stanford University School of

UMR 1068, Inserm, CRCM, BP30059, Cedex9, 27 Boulevard Leï Roure, Marseille 13273, 


\subsection{TCTP Is Upregulated in Prostate Cancer}

Although Prostate cancer (PC) is a deadly cancer with a rapidly increasing frequency in the western countries localized PC is usually treated with surgery and radiation (American cancer society: prostate cancer statistics http://www.cancer. org) (Jemal et al. 2010; Siegel et al. 2012). The importance of androgens for the initiation and progression of $\mathrm{PC}$ has been shown early in the twentieth century. Androgens bind to their specific receptors (AR) and are well known to supply the $\mathrm{PC}$ cell growth.

In 2004, a first study has shown that, while TCTP appears to be essential for prostate gland normal physiologic functions, its expression is increased in PC cells (Arcuri et al. 2004). The authors described TCTP as one of the main calcium binding protein in PC cells in which it regulates key processes like apoptosis and cellular differentiation. More recently, Gnanasekar et al. have examined if TCTP was a good potential new therapeutic target in androgen-dependent PC cells. Specifically, it has been demonstrated that silencing TCTP gene with a siRNA dramatically increases the androgen-sensitive PC cell death due to apoptosis (Gnanasekar et al. 2009). TCTP has further been identified as a novel androgen regulated gene whose expression is induced at both mRNA and protein level by androgens (Kaarbo et al. 2013).

\subsection{TCTP: A Promising Target in Castration-Resistant Prostate Cancer}

The first-line treatment for advanced or metastatic PC is the castration therapy consisting in androgen deprivation. Castration therapy cut off the supply of androgens that encourage PC growth (McLeod 2003; Theodore 2004). Despite the dramatic tumor regression that follows the castration therapy, the patients will ultimately become unresponsive and the prostate tumors will relapse within 1-3 years in a more aggressive castration-resistant mode (Fusi et al. 2004).

\subsubsection{TCTP Mediates Heat Shock Protein 27 Cytoprotective Function in CRPC}

In 2005, Rocchi et al. found that one of the most common genetic events in castration-resistant prostate cancer (CRPC) is the activation of heat shock protein 27 (Hsp27) expression (Rocchi et al. 2005). But the mechanism by which Hsp27 induces a multi-drugs resistance to CRPC tumors was unknown. Thus, in order to elucidate the pathways by which Hsp27 imposes its cytoprotective effect and find new therapeutic targets specific of CRPC tumors, Dr. Rocchi laboratory has 
screened all Hsp27 interactors using a two-hybrid system (Katsogiannou et al. 60 2014) and identified TCTP as a new Hsp27 protein partner (Baylot et al. 2012). 61 This work pinpoints for the first time TCTP as a potential therapeutic target in 62 CRPC (Baylot et al. 2012; Acunzo et al. 2014).

This study has demonstrated that TCTP protein level correlates with PC cells 64 aggressiveness. In castration-resistant (CR) prostate tumor cells, TCTP protein 65 expression is strongly increased compared to its expression in castration naïve PC 66 cells. Furthermore, a tissue microarray experiment performed on 211 clinical speci- 67 mens showed that TCTP is highly uniformly overexpressed in $75 \%$ of the CRPC 68 samples. These observations highlight its association with the aggressiveness of the 69 human disease. Additionally, no or weak TCTP expression has been detected in 70 normal or benign tissues, suggesting that targeting TCTP in human CRPC may 71 cause only weak undesirable toxicity in normal tissues.

Further mechanistic investigations showed that in castration-sensitive cells, 73 overexpressing Hsp27 is sufficient to increase TCTP protein level but not TCTP 74 mRNA level. Additionally, it has been demonstrated that Hsp27 is a direct upstream 75 regulator of TCTP and that this chaperone protects TCTP from its ubiquitination 76 and proteasomal degradation.

In CRPC cells, TCTP inhibition leads to cell viability reduction, cell cycle arrest, 78 and caspase-3-dependent apoptosis activation. Moreover, in castration naïve PC 79 cells stably overexpressing Hsp27, TCTP downregulation increases apoptosis via 80 caspase- 3 activation and enhances chemotherapy. These data show that TCTP 81 silencing suppresses the chemo-resistance of CRPC cells due to high Hsp27 levels 82 and suggest that TCTP is a mediator of Hsp27 cytoprotective function in CR pro- 83 state tumors. Furthermore, targeting TCTP in vivo with an antisense oligo- 84 nucleotide, developed by Dr. Rocchi laboratory (Baylot et al. Patent PCT10306447.3 85 2010), suppresses the growth of PC cell xenografts and significantly enhances chemo- 86 therapy activity upon systemic delivery. These findings open up the possibility for 87 using TCTP knockdown in combination with other established therapeutic approaches 88 to increase treatment efficacy in CRPC.

\subsubsection{TCTP and P53 in CRPC: "Neither Can Live While the Other Survives"}

Very interestingly, Baylot et al. also found that CR progression correlates with the 92 loss of the tumor suppressor P53. In a prior study, Amson et al. has shown that 93 TCTP and P53 are involved in a reciprocal negative-feedback loop in breast cancer 94 (Amson et al. 2012). But the role of P53 in the PC progression was still elusive. 95 Baylot et al. has demonstrated for the first time a link between TCTP, P53, and the 96 CR progression of PC (Fig. 13.1).

On one hand, after castration, the prostate tumors that have progressed from a 98 castration-sensitive state to a CR state overexpress TCTP and loose P53 expression. 99 


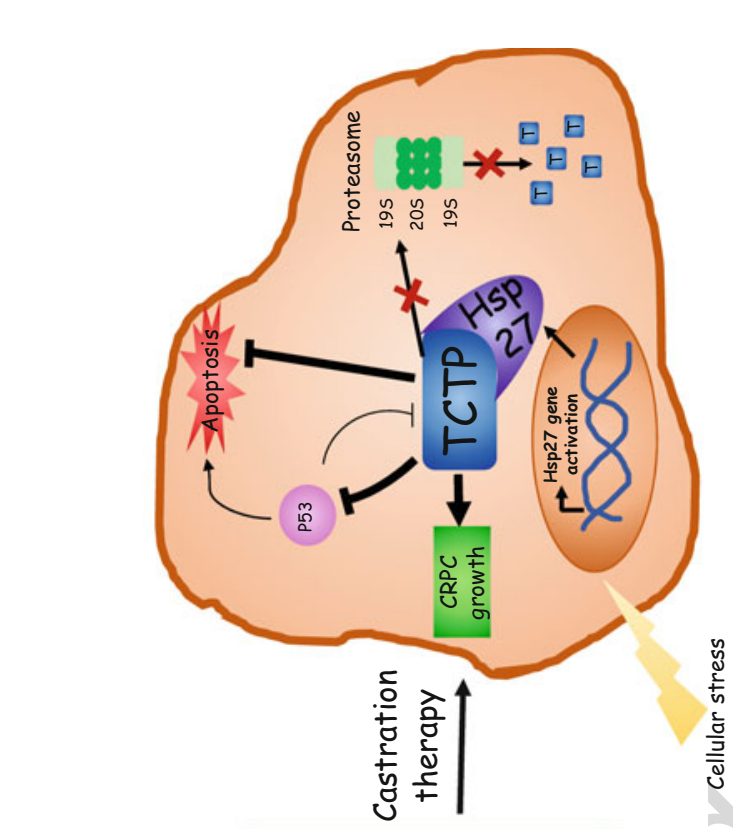

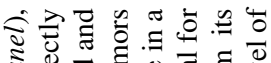

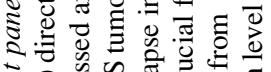

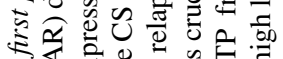

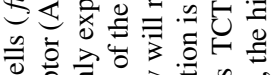

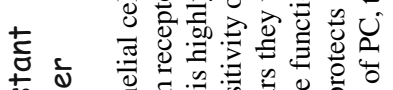

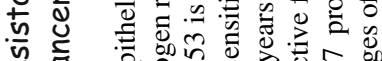
पु छु

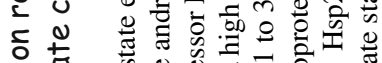

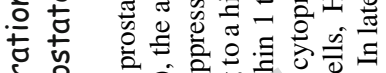
索立 西伦

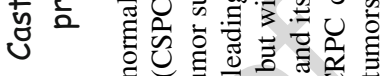
品主记

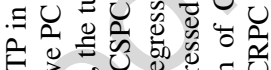
U. 응 녕

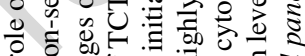

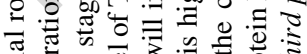

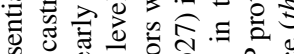
ชै $\leqq \subseteq$ उ

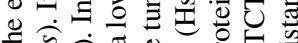

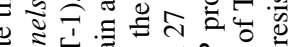

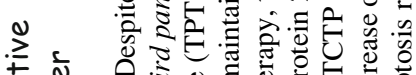

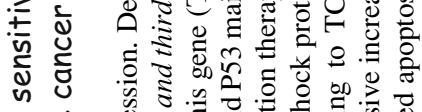
б.

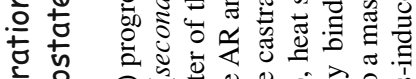

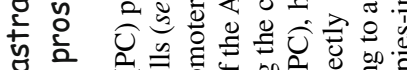

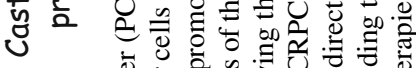

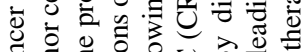
Uू

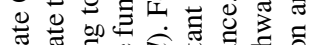

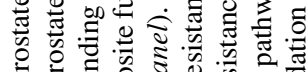

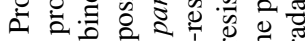
$\Xi \Xi$ 元

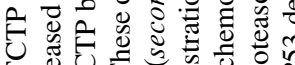
旸记

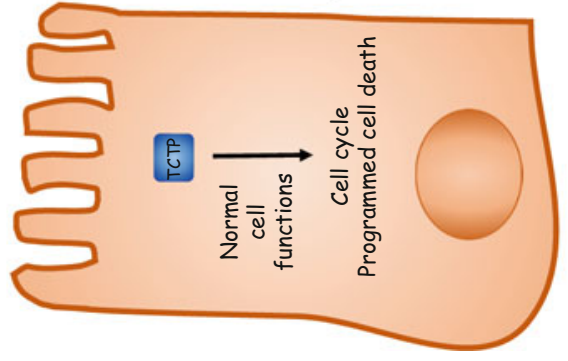

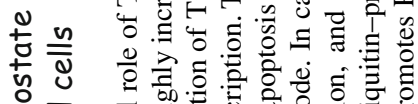

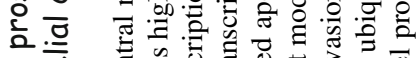

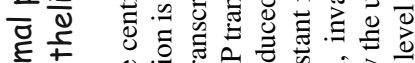

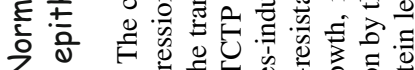
Z

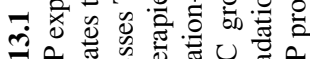

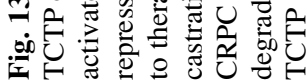


TCTP silencing using TCTP antisense oligonucleotide is able to restore P53 expres- 100 sion and function in CRPC tumors, suggesting that castration sensitivity is 101 directly linked to P53. On the other hand, P53 downregulation in castration- 102 sensitive PC cells significantly inhibits chemotherapy-induced apoptosis compared to 103 the control cells, suggesting an important link between P53 status and PC tumors 104 $\begin{array}{lr}\text { chemotherapy resistance. } & 105\end{array}$

These data show that TCTP is upregulated in CRPC tumors leading to the loss of 106 P53 expression and function together with castration- and chemo-therapies resis- 107 tance. This work importantly highlights the crucial role of TCTP/P53 axis in 108 CR progression of $\mathrm{PC}$.

\subsubsection{Development of a TCTP Antisense Oligonucleotide for Clinical Applications}

As mentioned above, Dr. Rocchi laboratory has developed a TCTP inhibitor that 112 can be used for human therapy and has screened by gene walk all antisense oligo- 113 nucleotide (ASO) sequences targeting TCTP full-length mRNA (Karaki et al. 114 2017). Initially, 28 ASOs have been designed. Finally, three ASO lead sequences, 115 that potently inhibited TCTP expression, have been furthered examined for their 116 ability to affect CRPC cells and tumor growth. Thus, it has been reported that 117 TCTP-ASOs enter to the cells via macropinocytosis, increased caspase-3-depen- 118 dent apoptosis, blocked cell cycle, and enhanced chemotherapy in CRPC cells 119 in vitro. And, consistent with these in vitro data, systemic administration of 120 TCTP-ASOs in immunocompromised mice suppressed CRPC tumor growth and 121 also significantly enhanced castration and chemotherapy activities in vivo. Addi- 122 tionally, TCTP-ASO treated mice showed a significant decrease of Ki-67 levels, a 123 proliferation marker, compared to the control group. Moreover, possible toxic 124 effects resulting from oligonucleotide administration have been checked. The 125 animals did not show any change neither in their behavior, nor in their body weight 126 during the experiment. Furthermore, treated mice did not show any signs for 127 hepatic damage, since their aspartate aminotransferase (AST) and alanine amino- 128 transferase (ALT) levels were within the range of normal values, and they didn't 129 show significant difference compared to the control group. Finally, no sign of 130 renal damage was observed, since the creatinine levels were normal and the bio- 131 chemical analyses of urine were normal for the tested parameters. 


\subsection{Discussion}

Taken together, these studies demonstrate that TCTP has a crucial role in the different stages of PC malignant progression from the tumor initiation to the multi-drugs resistant stage of the disease.

Arcuri et al. were the first to investigate TCTP expression and function in human prostate normal and cancer tissues. The authors have notably found that TCTP is the most highly expressed calcium binding protein in the human prostate cancer cells. Subsequent studies from different laboratories confirmed that TCTP is upregulated in early stages of the disease, in which the prostate tumor growth is fueled by androgens (Gnanasekar et al. 2009; Kaarbo et al. 2013). TCTP has since been identified as an androgen-regulated gene (Kaarbo et al. 2013). Furthermore, TCTP has been reported to be causal for the resistance to androgen withdrawal and chemotherapy in PC (Baylot et al. 2012; Acunzo et al. 2014). This work has validated, using 211 clinical specimens, that TCTP is slightly over-expressed in the castration naïve specimens compared to normal specimen, confirming his implication in PC initiation, and that its expression is abolished upon castration therapy. Most importantly this study has shown for the first time, that TCTP is highly overexpressed in multi-drugs resistant prostate tumors and metastases, pinpointing TCTP as a key protein in the late stages of PC in which the tumors grow in a castration-resistant mode (CRPC). Currently, there is no effective therapy for patients with CRPC and existing novel therapies only have a modest impact on the overall survival of these patients (McKeage 2012; de Bono et al. 2010). Clearly, novel approaches were required to effectively treat CRPC, in particular new strategies that identify the molecular mechanisms by which CRPC becomes chemoresistant, as well as the identification of novel therapeutic targets that could improve clinical outcome. Thus, identifying TCTP as a new therapeutic target for the treatment of CRPC represents a major advance in the field. Additionally, altogether these findings also strongly suggest that TCTP is highly prognostic in human PC.

A TCTP inhibitor has been developed by Dr. Rocchi laboratory (Baylot et al., Patent PCT10306447.3; 2010) for clinical applications. Recent results showed that the TCTP inhibitor can suppress CRPC tumor growth and enhance castration- and chemo-therapies in vitro and in vivo. The stability, biodisponibility, and delivery improvement of this TCTP inhibitor for human treatment is currently under investigation in Dr. Rocchi laboratory and represents today a great hope for the patients with CRPC.

\section{References}

Acunzo J, Baylot V, So A, Rocchi P (2014) TCTP as therapeutic target in cancers. Cancer Treat Rev 40(6):760-769

Amson R, Pece S, Lespagnol A, Vyas R, Mazzarol G, Tosoni D et al (2012) Reciprocal repression between P53 and TCTP. Nat Med 18:91-99 
Arcuri F, Papa S, Carducci A, Romagnoli R, Liberatori S, Riparbelli MG et al (2004) Trans- 173 lationally controlled tumor protein (TCTP) in the human prostate and prostate cancer cells: 174 expression, distribution, and calcium binding activity. Prostate 60:130-140 175

Baylot V, Andrieu C, Acunzo J, Katsogiannou M, Rocchi P (2010) Nucleic acid targeting TCTP 176 for use in the treatment of cancer. Patent PCT10306447.3 177

Baylot V, Katsogiannou M, Andrieu C, Taieb D, Acunzo J, Giusiano S et al (2012) 178 Targeting TCTP as a new therapeutic strategy in castration-resistant prostate cancer. 179 Mol Ther 20(12): 2244-2256 180

de Bono JS, Oudard S, Ozguroglu M, Hansen S, Machiels JP, Kocak I et al (2010) Prednisone plus 181 cabazitaxel or mitoxantrone for metastatic castration-resistant prostate cancer progressing after 182 docetaxel treatment: a randomised open-label trial. Lancet 376:1147-1154 183

Fusi A, Procopio G, Della Torre S, Ricotta R, Bianchini G, Salvioni R et al (2004) Treat- 184 ment options in hormone-refractory metastatic prostate carcinoma. Tumori 90:535-546 185

Gnanasekar M, Thirugnanam S, Zheng GX, Chen AS, Ramaswamy K (2009) Gene silencing of 186 translationally controlled tumor protein (TCTP) by siRNA inhibits cell growth and induces 187 apoptosis of human prostate cancer cells. Int J Oncol 34:1241-1246 188

Jemal A, Siegel R, Xu J, Ward E (2010) Cancer statistics, 2010. CA Cancer J Clin 60:277-300 189

Kaarbo M, Storm M, Qu S, Wæhre H, Risberg B, Danielsen HE et al (2013) TCTP is an andro- 190 gen regulated gene implicated in prostate cancer. PLoS One 8(7):e69398 191

Karaki S et al (2017) Lipid-oligonucleotide conjugates improve cellular uptake and efficiency of 192 TCTP-antisense in castration-resistant prostate cancer. J Control Release 258:1-9 193

Katsogiannou M, Andrieu C, Baylot V, Baudot A, Dusetti NJ, Gayet O et al (2014) The functional 194 landscape of Hsp27 reveals new cellular processes such as DNA repair and alternative splicing and 195 proposes novel anticancer targets. Mol Cell Proteomics 13(12):3585-3601 196

McKeage K (2012) Docetaxel: a review of its use for the first-line treatment of advanced castration- 197 resistant prostate cancer. Drugs 72:1559-1577 198

McLeod DG (2003) Hormonal therapy: historical perspective to future directions. Urology 61:3-7 199

Rocchi P, Beraldi E, Ettinger S, Fazli L, Vessella RL, Nelson C et al (2005) Increased Hsp27 after 200 androgen ablation facilitates androgen-independent progression in prostate cancer via 201 signal transducers and activators of transcription 3-mediated suppression of apoptosis. 202 Cancer Res 65:11083-11093 203

Siegel R, Naishadham D, Jemal A (2012) Cancer statistics, 2012. CA Cancer J Clin 62:10-29 204 Theodore C (2004) Hormone therapy for prostate cancer. Bull Cancer 91:69-74 205 